\title{
Correction to: Long-term results achieved by guideline-based stage-dependent management of anal cancer in a non-HIV population
}

\author{
Bernhard Fankhaenel ${ }^{1}$ (D) J Joerg Zimmer ${ }^{2}$ - Dorothea Bleyl ${ }^{3}$ - Eric Puffer ${ }^{4} \cdot$ Andreas Schreiber $^{2} \cdot$ Thomas Kittner $^{5}$. \\ Helmut Witzigmann ${ }^{1} \cdot$ Sigmar Stelzner ${ }^{1}$
}

Published online: 20 August 2020

(C) Springer-Verlag GmbH Germany, part of Springer Nature 2020

\section{Correction to: International Journal of Colorectal Disease (2019) 34:1895-1905 https://doi.org/10.1007/s00384-019-03396-x}

The authors of the article mentioned above found out errors on the Table 4 concerning the number and survival rates of patients with incomplete radiotherapy.

Lines $13-15$ in the 8 th paragraph of the Results section now reads as follows:

"A trend towards longer survival was found if chemoradiotherapy was performed completely (77.7 vs. $62.7 \%$, Table 4$). "$

The online version of the original article can be found at https://doi.org/ 10.1007/s00384-019-03396-x

Sigmar Stelzner

stelzner-si@khdf.de

1 Department of General and Visceral Surgery, Dresden-Friedrichstadt General Hospital, Teaching Hospital of the Technical University of Dresden, Friedrichstr. 41, 01067 Dresden, Germany

2 Department of Radiation Therapy, Dresden-Friedrichstadt General Hospital, Teaching Hospital of the Technical University of Dresden, Friedrichstr. 41, 01067 Dresden, Germany

3 Department of Medical Oncology, Dresden-Friedrichstadt General Hospital, Teaching Hospital of the Technical University of Dresden, Friedrichstr. 41, 01067 Dresden, Germany

4 Department of Pathology, Dresden-Friedrichstadt General Hospital, Teaching Hospital of the Technical University of Dresden, Friedrichstr. 41, 01067 Dresden, Germany

5 Department of Radiology, Dresden-Friedrichstadt General Hospital, Teaching Hospital of the Technical University of Dresden, Friedrichstr. 41, D-01067 Dresden, Germany 
The Table with correct figures in the last two lines is shown as follows:

Table 4 Overall survival

\begin{tabular}{|c|c|c|c|c|c|}
\hline & Number & $\begin{array}{l}\text { Survival rate } \\
\text { at } 5 \text { years }\end{array}$ & $\begin{array}{l}95 \% \text { confidence } \\
\text { interval }\end{array}$ & Events & $P$ values \\
\hline All & 103 & 73.1 & $64.5 \ldots 81.7$ & 27 & \\
\hline \multicolumn{6}{|l|}{ Localisation } \\
\hline Canal & 66 & 67.5 & $55.9 \ldots 79.1$ & 21 & \multirow[t]{2}{*}{0.071} \\
\hline Margin & 37 & 83.2 & $70.9 \ldots 95.5$ & 6 & \\
\hline \multicolumn{6}{|l|}{ T-category } \\
\hline $1-2$ & 76 & 82.7 & $74.1 \ldots 91.3$ & 13 & \multirow[t]{2}{*}{$<0.001$} \\
\hline $3-4$ & 27 & 44.9 & $25.1 \ldots 64.7$ & 14 & \\
\hline \multicolumn{6}{|l|}{ N-category } \\
\hline 0 & 66 & 78.2 & $68.0 \ldots 88.4$ & 14 & \multirow[t]{2}{*}{0.134} \\
\hline Positive & 37 & 64.2 & $48.5 \ldots 79.9$ & 13 & \\
\hline \multicolumn{6}{|l|}{ Grading $^{\mathrm{a}}$} \\
\hline $1-2$ & 88 & 76.7 & $67.7 \ldots 85.7$ & 20 & \multirow[t]{2}{*}{0.003} \\
\hline 3 & 12 & 40.0 & $11.4 \ldots 68.6$ & 7 & \\
\hline \multicolumn{6}{|l|}{$\mathrm{SCC}^{\mathrm{b}}$} \\
\hline $\operatorname{Normal}(<=1.5 \mathrm{ng} / \mathrm{ml})$ & 49 & 75.5 & $63.3 \ldots 87.7$ & 12 & \multirow[t]{2}{*}{0.702} \\
\hline Elevated $(>1.5 \mathrm{ng} / \mathrm{ml})$ & 29 & 70.2 & $52.8 \ldots 87.6$ & 8 & \\
\hline \multicolumn{6}{|l|}{ Radiotherapy $^{\mathrm{c}}$} \\
\hline All & 82 & 73.6 & $63.8 \ldots 83.4$ & 21 & \multirow{3}{*}{0.143} \\
\hline Complete & 63 & 77.7 & $66.4 \ldots 87.6$ & 14 & \\
\hline Incomplete & 19 & 62.7 & $40.7 \ldots 84.7$ & 7 & \\
\hline
\end{tabular}

Patients with synchronous distant metastases (M1) excluded

${ }^{\mathrm{a}}$ Missing for 3 patients

${ }^{\mathrm{b}}$ Missing for 25 patients

${ }^{\mathrm{c}}$ Curative and additive intent only

Publisher's note Springer Nature remains neutral with regard to jurisdictional claims in published maps and institutional affiliations. 